\title{
The design of liquor cellar monitoring system based on Windows CE
}

\author{
Ju Jinwu ${ }^{1, a}$, Wang Lanying ${ }^{1, b}$ \\ ${ }^{1}$ School of Computer Science, Sichuan University of Science and Engineering, Zigong, Sichuan, \\ China \\ ajjwmail@163.com, ${ }^{b}$ jjwly@163.com
}

Keywords: Windows CE, Liquor cellar, Monitoring, Wireless, Terminal.

\begin{abstract}
There is a great influence for output and quality of fermentation process on liquor cellar environmental factors, with the rapid development of the liquor industry, production scale and variety of fermentation products are increasing, it is very important to carry out intelligent monitoring of fermentation process. This article introduces the design of liquor cellar monitoring system based on Windows CE operating system, the system based on a modular structure, the design and implementation of wireless collection node and intelligent monitoring terminal, according to the specific situation on site, rapidly constructing the suitable monitoring platform, and strong extendibility.
\end{abstract}

\section{Introduction}

Liquor fermentation is the most important part of the entire liquor production process, it is a complex process of microbial metabolism. With the rapid development of the liquor industry, production scale and variety of fermentation products are increasing, so the implementation of intelligent monitoring on the fermentation process, and real-time effective control and optimization be imperative. In most of the liquor business in our country, the staff mainly carrying on the traditional detection device with simple structure, specific parameters in the fermentation process of manual reading. Backward measuring device, the error of manual reading and measure the parameters of non-real time caused the information acquisition with poor reliability, limited amount of information, information lag etc.

The liquor cellar monitoring system completes to many cellar in microorganism fermentation environment parameter gathering, collects the data, supply the suitable liquor cellar control system, controls the liquor cellar external environment essential factor, causes the liquor cellar the fermentative process to be at the optimum condition, enhances the liquor cellar to produce the liquor efficiency. In the entire liquor cellar monitor system, between the cellar ponds are at dispersible conditions, each liquor cellar is realizes its data collection through a wireless gathering node, several wireless gathering nodes composed to wireless sensor network, monitors the network intelligence terminal based on the Windows CE embedded operating system main engine, completes with each wireless gathering node to wireless communication. The intelligent terminal and the gathering node all based on CC1101 the Zigbee chip realization wireless communication, in the node runs the wireless sensor network protocol, all nodes structure the wireless sensor network, intelligent terminal through gathering node realization to overall system data collection. In Windows CE embedded system, realized the CC1101 chip driver, uses in supporting running on the upper wireless sensor network protocol.

\section{Liquor cellar overall structure of the monitoring system}

The environment of liquor fermentation cellar is complex, it's a great challenge for fermentation process data in real time gathering. Liquor cellar monitoring system full utilizing the comprehensive perception technology of the networking, communications technology, information intelligent analysis and control technology, according to the above technology achieve intelligent Sense for liquor cellar fermentation process. The overall architecture of the system is shown in Fig.1. 


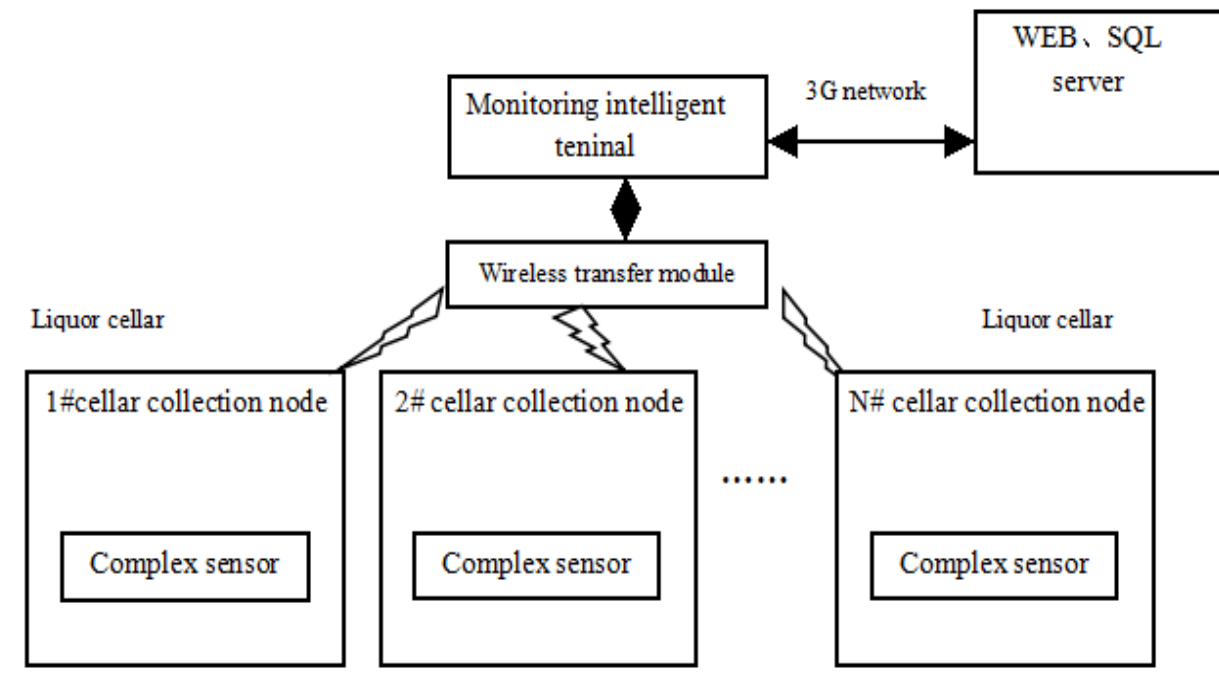

Fig. 1 overall construct of the monitoring system

Liquor cellar monitoring system is constructed by 3 layer mode based on networking, the perception layer is composed by the cellar collections node and composite sensors. Network layer includes wireless transmission module and monitoring intelligent terminals. Application layer is consists of WEB programming and SQL database servers. Cellar need to detect environmental factors including temperature and humidity, concentration of alcohol, dissolved oxygen concentration, $\mathrm{PH}$ values, $\mathrm{CO} 2$ concentrations and temperature data. For easy to operate, combining these sensors into a compound, cellar gathering node for each sensor's measurement data, then by wireless transferring , through the wireless transmission module to upload the data, sent to monitor intelligent terminal, on the one hand the monitor intelligent terminal can display these data, on the other hand the data uploaded to the WEB and SQL servers, supply to application level programs.

According to the liquor cellar data acquisition nodes requirements of functional and properties, the node structure design as shown in Fig.2. The system consists of a MCU unit, RF unit, sensor unit, power \& voltage conversion unit, a charging unit. All units are using low power components. For large power consumption units, such as MCU and RF transceiver units are used with sleep function element. Wireless sensor module MCU using STC11L04's STC chip, RF transceiver unit uses the CC1101 chip of TI company.

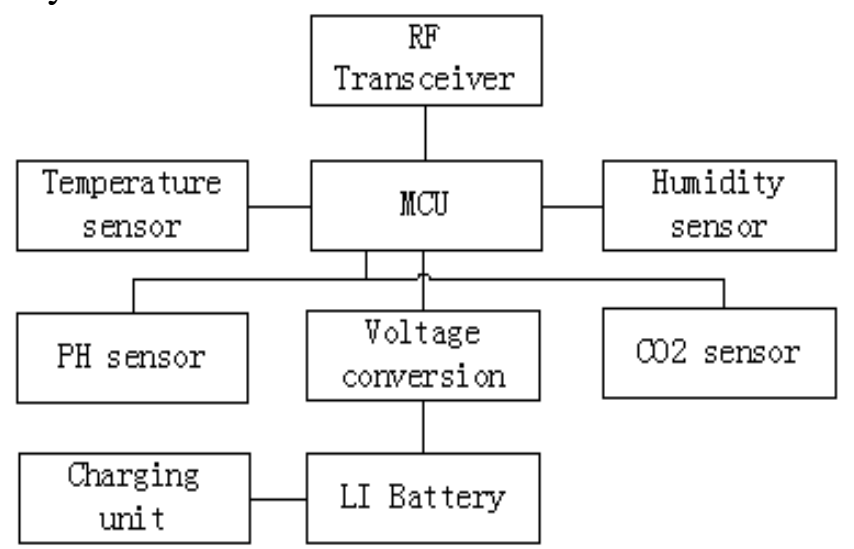

Fig.2. liquor cellar construct for gathering wireless node

The important module of the network layer is liquor cellar intelligent terminal, the terminal carry out information access and data transmission. The variety of environmental factors in complexity liquor collar decides the variety of information transmission. Network transmission should be according to the specific circumstances of the site adopt flexible forms of structure, in generally adopting multi-input and multi-output for multi-path transmission. The structure of the intelligent terminal as shown in Fig.3, the intelligent terminal with ARM processor as the main processor, ARM processor is a kind of embedded processor, and has the advantages of high performance, low cost and low power consumption. S3C6410 is a 32-bit processor and operating frequency up to a maximum of $800 \mathrm{MHz}$, built a large number of hardware components inside, the 64/32 bit bus structure, including 
16kb instruction / data cache, MMU memory management device, digital camera interface, video coding decoder, ac-97 audio interface, IIC, SPI serial bus, 4 UART channels, real-time clock (RTC), ADC, USB host and device interface, LCD controller, general purpose I / O port and so on, are very convenient to use, greatly simplified the structure design of pits intelligent terminal.

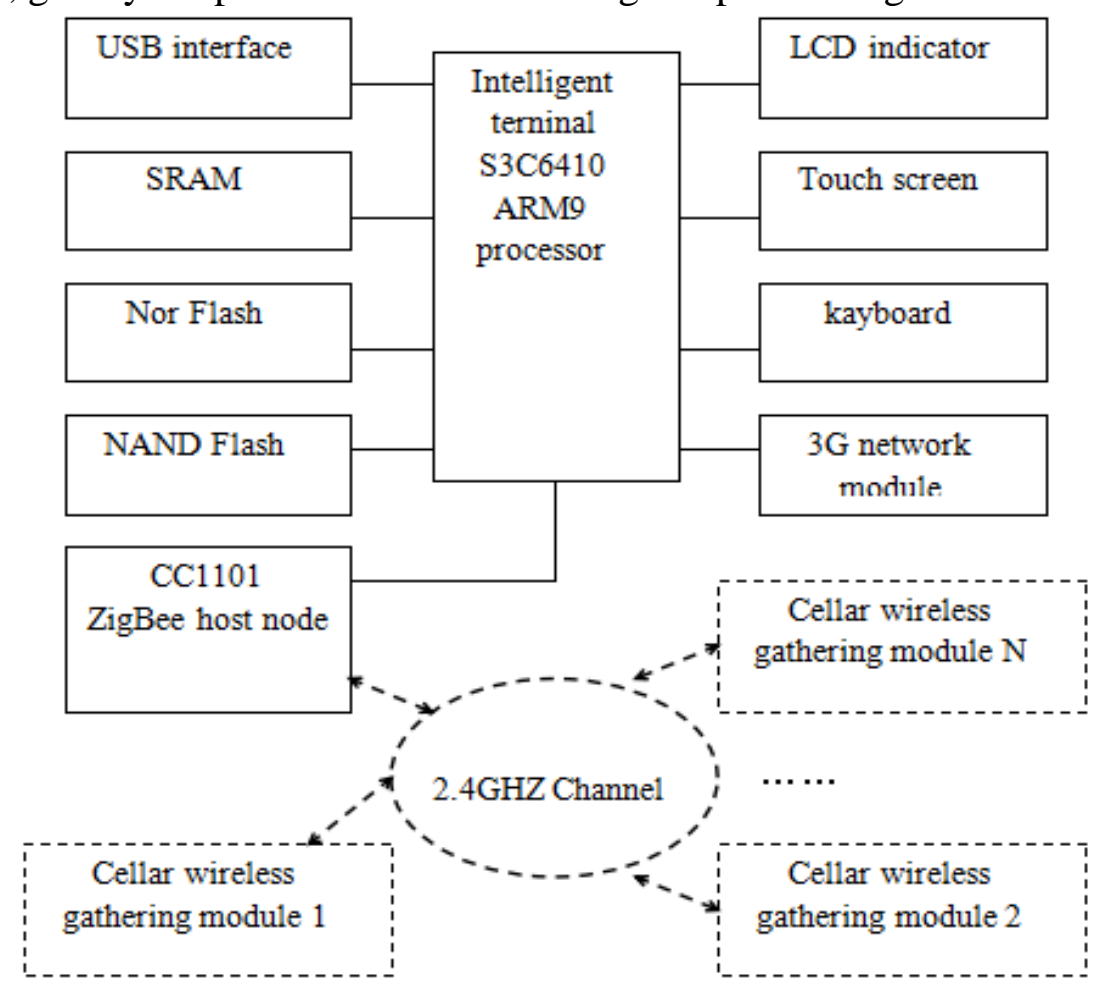

Fig.3 .liquor cellar intelligent terminal structure

\section{The software design of the monitoring system}

Intelligent terminal monitoring as a center of intelligent monitoring system, main function is: read various environment parameters of the wireless data collecting module, data stored on the SD card, and upload to the web and SQL server, supply program layer use; by using intelligent control algorithm to deal with the received data, and can receive the control command of the computer; own independent man-machine interface can through the LCD, touch screen and keyboard for live data display and operation.

The main program includes: wireless module of communication, LCD and keyboard of human-computer interaction, the date and time of reading, data of on-site storage and main program flow as shown in Fig.4. Intelligent monitoring terminal design of Windows CE based on the embedded operating system, which will be ported to run on S3C6410, RC3 version 6. In the Windows CE operating system to design the cc1101 driver, system can access the cc1101, cc1101 configuration, and wireless communication.

loading the CC1101 driver and the equipment initialize. According to the needs of the network composed wireless sensor, setting the working frequency, the CC1101 channel, working mode.

Starting the data receiving thread, complete with the liquor cellar data acquisition node communication, receiving data uploaded.

LCD displayed the field data. The system is equipped with a large screen LCD liquid crystal display, real-time data can be displayed by the cellar.

Starting the data upload thread, through the 3G network, complete the data uploaded to the WEB server, SQL database. Data servers for the upload process, implementation based on HTTP protocol, the ARM processor by DTU and the web server to realize the network connection, data upload task program through build the underlying HTTP get data packets, to the web server to upload data, again by the procedure of web writing SQL database server in. 


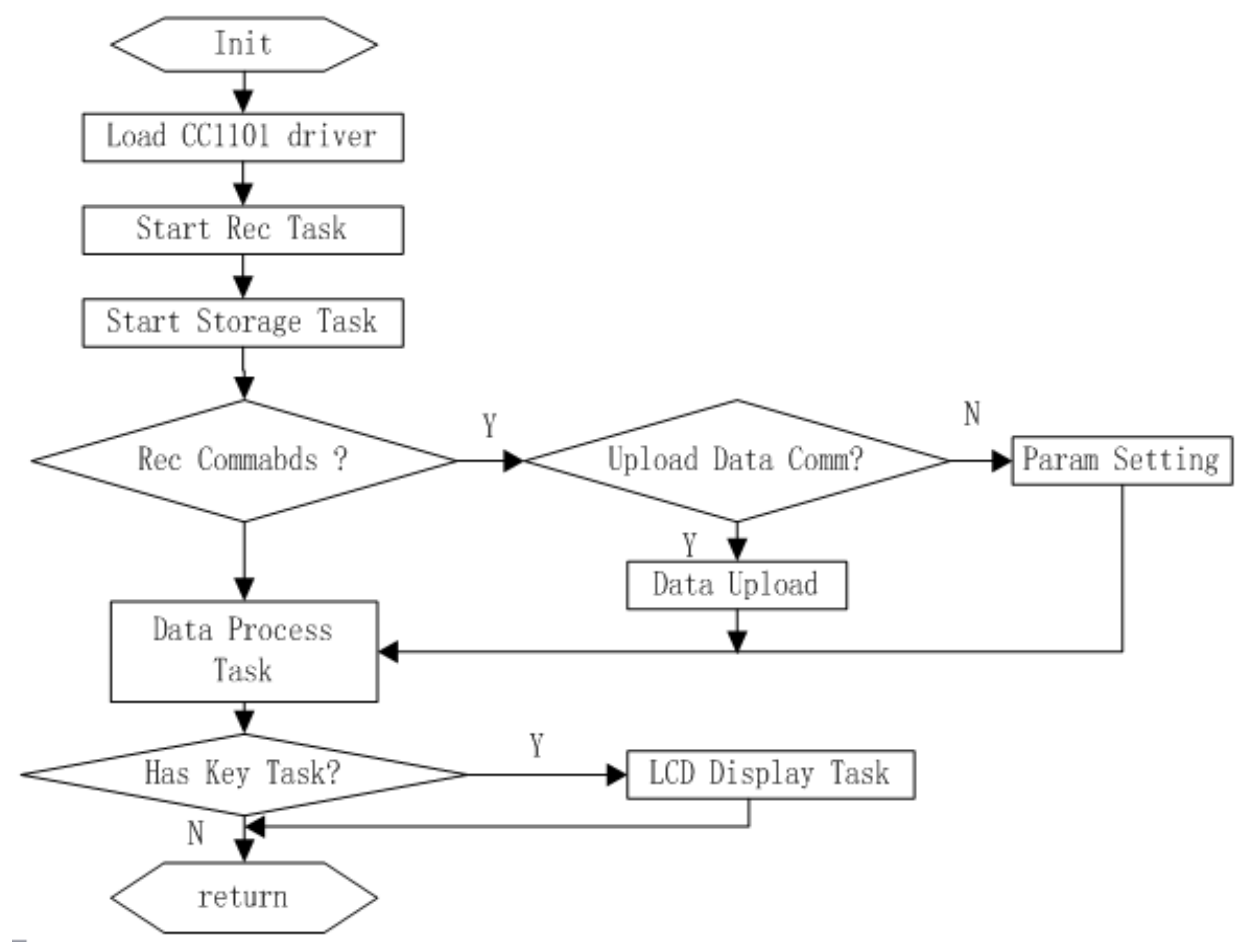

Fig.4. Flow chart of the main program

\section{The monitoring system operation results}

During the fermentation in liquor cellars is very complicated, in different cellars, fermentation condition is not the same. As temperature, for example, fermentation will lead to different locations of the temperature difference, monitoring system in the cellar, hierarchically arranged on the upper, middle and lower temperature sensors, Xiaoqu liquor fermentation cycle generally a week or so, once every 10 minutes to measure pit temperature, the obtained temperature data to curve show that fig. 5 shows:

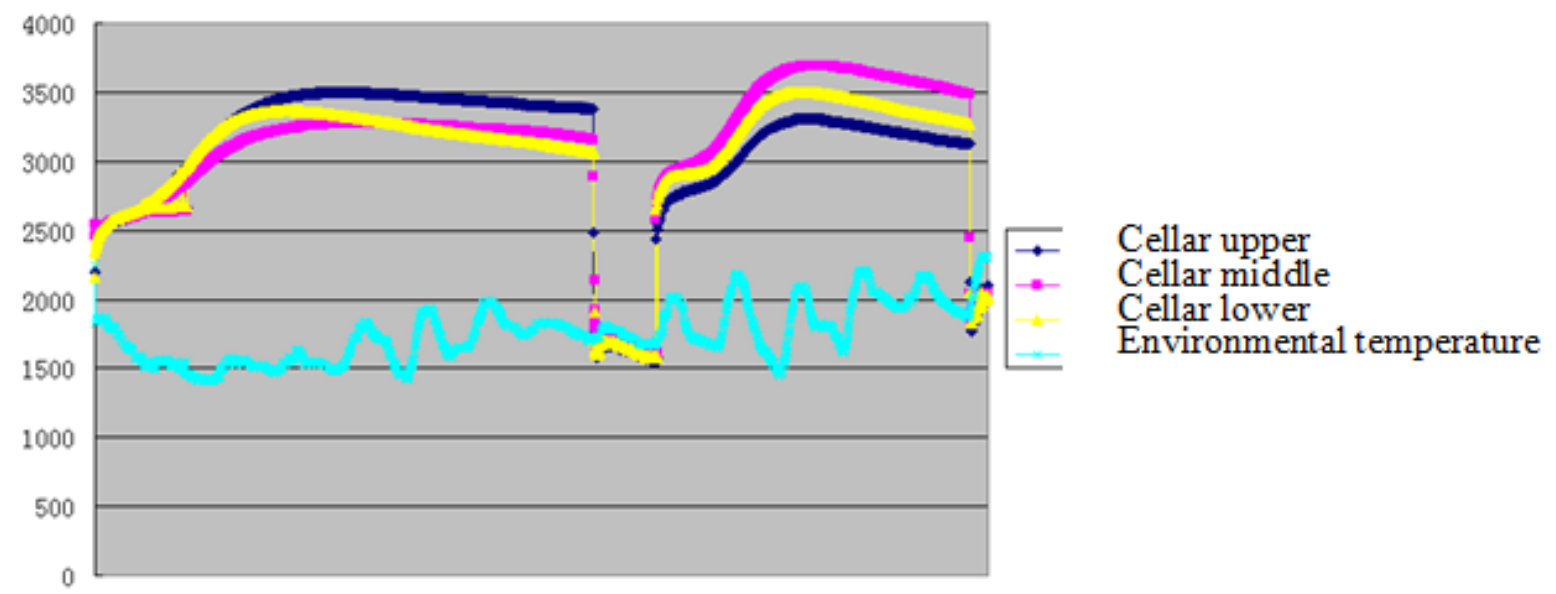

Fig.5. The temperature curve in liquor cellars

Temperature curve of the horizontal axis is time, the vertical axis is the temperature, includes four curves, respectively is liquor cellars the external environment temperature and upper, middle and lower three layers of three temperature data. As can be seen from Figure 5, pits of internal temperature change trend, first with fast rise time, indicating that fermentation process to be more violent, and then the temperature dropped slowly, indicating that fermentation had gradually become stable. Fig. 5 shows the two fermentation cellars, showing the law. The lower the ambient temperature curve has more than the internal temperature is low, the fluctuation reflects the diurnal and night temperature variation environment. 


\section{Conclusion}

This paper introduces a based on Windows CE operating system design of liquor cellar monitoring system for monitoring of liquor cellars environmental factors, temperature changes in the liquor cellar fermentation were studied. The design of the system based on Internet network three layer structure, through the use of the modular and structured design thought, in reducing the difficulty of the design at the same time, stability and reliability of the monitoring system has been significantly enhanced, at the same time, the system maintenance becomes more simple. Monitoring system in Sichuan Hong Mao wine factory of the experimental operation, the running results show: data collection is accurate, reliable data transmission, better completed the monitoring task. This paper briefly analyzes the hardware structure and software function of the intelligent terminal. Introduced the design method of the system. For the design and development of wireless sensor network system has practical reference value.

\section{Acknowledgement}

Foundation item: Artificial Intelligence Key Laboratory Project of Sichuan Province (2013RZY03), Enterprise Informatization and the Internet of Things Measurement and Control Technology Key Laboratory Project(2014WYY01).

\section{References}

[1] LIN Yi-duo,GAO De-yun,LIANG Lu-lu ,et al. Design and implementation of MAC protocol for wireless sensor network based on ARM[J]. Journal of Computer Applications, 2010, 30(5): 1145-1148

[2] TONG Dong-bing,CAI Le-cai,GAO Xiang,LI Hong-chan. Design and Implementation of the Multi-Serial Gateway Based on Embedded[J]. Journal of Sichuan University of Science \& Engineering(Natural Science Edition). 2009,22(5):44-46

[3] LIAO Huan-zhu,FANG Kang-ling,Cao Jing. 12-Lead ECG design based on S3C6410 and WinCE 6.0[J]. Journal of Computer Applications,2010,A01:331-333

[4] WANG Jun-he,GAO Fei,WU Jie-lin. Point-to-Point Wireless Data Acquistition Based on CC1100[J]. Computer Science,2011,38(10):212-214

[5] GUO Xue-lian,Leng Jian-zhu,He Xiang-zhi. Design of Ethernet Access for Serial Devices Based on S3C44B0X and UClinux[J]. Journal of SICHUAN university of science \& engineering(Natural science edition),2008,21(5):29-32

[6] FU Yong-hong,ZHAO Pan. Design of I2C Bus Driver Based on S3C2410[J]. Journal of Sichuan University of Science \& Engineering(Natural Science Edition),2009,22(3):43-46 